\title{
Study on Image Retargeting Methods in View of Importance-Map
}

\author{
Zijuan Zhang ${ }^{1}$, Baosheng Kang ${ }^{*}$ and Shunye Wang ${ }^{2}$ \\ ${ }^{1}$ School of Information Science and Technology, Northwest University \\ $X i^{\prime}$ an 710127, China \\ * School of Information Science and Technology, Northwest University \\ Xi'an 710127, China \\ ${ }^{2}$ Department of Computer Science and Technology, Langfang Teachers University, \\ Langfang 065000, China \\ ${ }^{1}$ E-mail: bluesky1023@126.com, ${ }^{*}$ E-mail: bskang@163.com(Corresponding author), \\ ${ }^{2}$ shunyew@163.com
}

\begin{abstract}
This paper analyzes the characteristics of the state-of-the-art technologys with regard to content-aware image retargeting in detail, contrasts the merits and demerits between diverse technologys, and lists the performance and effect about each method. The primary processes of content-aware image retargeting are divided into two steps: first step is the recognition of image importance-map, which is generally generated by the combination of image gradient and visual significance, indicates that human eyes generates diverse sensitivity of watching different area in image; Current papers with respect to content-aware image retargeting primarily centers on the resizing method in the second step. In the light of the traits of different ways, this article subdivided the technologys into three categories: on account of seam-carving, image-deformation and multi-operator. At last, we give the final qualitative analysis about the three algorithms mentioned above.
\end{abstract}

Keywords: Retargeting, Importance-map,Seam-carving, Deformation, Multi-operatior

\section{Introduction}

In retargeting processes, the kernel of the content-aware image retargeting technology is that trying to maintain major object areas with the higher importance-degree unchanged and changing the non-trivial areas with lower importance-degree in order to fit the target displayer's size. In the light of the traits of different ways, this paper subdivided the technologies into three categories: on account of seam-carving, image-deformation and multioperator.

\section{The Retargeting Method on Account of Seam-Carving}

The retargeting method of seam-carving that has been presented by Avidan and Shamir [1] is also known as Backward-algorithm. Here, seam-carving is an image retargeting method that by continuously deleting or inserting seams which cut through the entire image achieves the reducing or amplying of the image. The breadth of the seam is only one pixel, if deleting the seam, then the image size will be smaller, on the contrary if inserting the seam, the picture will be larger. Handling the vertical(horizontal) seams will vary the width(height) of the image. For the sake of keeping the principal character of the image feature, the method of the seam-carving deletes or inserts the seam with minimal energy each time. The seam's energy 
is known as the sum of the pixels' energy in the seam. In the reference [1], the importancemap of the pixels is generated by gradient. Let $I$ be an image with size $n * m$ and define a vertical seam as :

$S^{v}=\left\{S_{i}^{v}\right\}_{i=1}^{m}=\left\{(i, x(i)\}_{i=1}^{m}\right.$, s.t. $\forall i,|x(i)-x(i-1)| \leq 1$

In above equation, $x(i)$ refers to a mapping : $x[1, \cdots, m] \rightarrow[1, \cdots, n]$. In other words, a vertical seam should be an 8-connected route of pixels from top to bottom in the image, including one, and just one, pixel in every row in the image. In the same way, a horizontal seam should be :

$S^{h}=\left\{S_{j}^{h}\right\}_{j=1}^{n}=\left\{(y(j), j\}_{j=1}^{n}\right.$, s.t. $\forall j,|y(j)-y(j-1)| \leq 1$

In this equation, $y(j)$ refers to a mapping : $y[1, \cdots, n] \rightarrow[1, \cdots, m]$. And likewise, a horizontal seam should be an 8 -connected route of pixels from left to right in the image, including one, and just one, pixel in every column in the picture.

Considering the energy function $e$, we are able to define a seam's cost as : $E(s)=\sum_{i=1}^{m} e(i, x(i))$, We search the optimum seam $s^{*}$ that can minimize the seam's cost :

$S^{*}=\min _{s} E(s)=\min _{s} \sum_{i=1}^{m} e(i, x(i))$

In the reference [1], the optimum seam can be searched applying dynamic programming algorithm. Firstly, the algorithm is traversing the picture totally from the second row to the final row and calculating the accumulative minimum energy $M$ for all feasible connecting seams for every entry $(i, j)$ :

$M(i, j)=e(i, j)+\min (M(i-1, j-1), M(i, j-1), M(i+1, j-1))$

In the references [3, 8, 9-11], the Backward algorithm is improved ulteriorly. In our experiment, the Backward algorithm is improved by adding some energy. We produce new standard due to the original method selects to delete the seam of the least amount of pixels' energy from the picture, neglecting energy that is introduced into the resized image.

The reference [3] presents new method of seam-carving, the algorithm increases the energy that is generated by new neighboring pixels which is produced by deleting pixels. As shown in Figure 1, deleting vertical seam can introduce three types of new neighboring pixels.

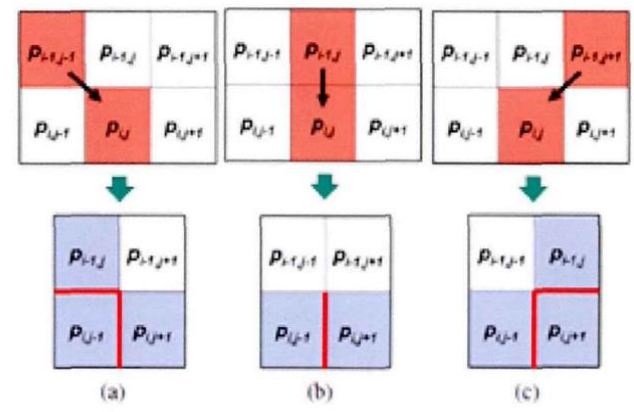

Figure 1. New Energy Generated by New Neighboring Pixels( Red Lines) 
With respect to each of the above three possible situations, we refers to a cost respectively as: $C_{L}(i, j) 、 C_{U}(i, j)$, and $C_{R}(i, j)$ :

$$
\begin{aligned}
& \text { (a) } C_{L}(i, j)=|I(i, j+1)-I(i, j-1)|+|I(i-1, j)-I(i, j-1)| \\
& \text { (b) } C_{U}(i, j)=|I(i, j+1)-I(i, j-1)|+|I(i-1, j+1)-I(i-1, j-1)| \\
& \text { (c) } C_{R}(i, j)=|I(i, j+1)-I(i, j-1)|+|I(i-1, j)-I(i, j+1)|
\end{aligned}
$$

In this equation, $I(i, j)$ is the pixel $(i, j)$ 's color value. Therefore, with respect to vertical seams, each costing $M(i, j)$ is refreshed applying the following regulation:

$$
M(i, j)=e(i, j)+\min \left\{\begin{array}{c}
M(i-1, j-1)+C_{L}(i, j) \\
M(i, j-1)+C_{U}(i, j) \\
M(i+1, j-1)+C_{R}(i, j)
\end{array}\right.
$$

Reference [8] has presented the algorithm in regard to continuous seam-carving algorithm. This algorithm has found the number of seams that surpass the amount which should be remored or inserted, and has made the seams undergo a certain percentage of deleting(inserting) amount, that is called resampling density. In this method, the deleting (inserting) amount of seams is in proportion to their energy. The reference [9] can delete more than one pixel and execute the function that shift the mainbody object, therefore this method is also known as Shift-map algorithm. The reference [10] considers that deleting or inserting the neighboring seams concurrently may break the overall visual effect. Hence if deleting the seams, it may increase the deleting seam energy to adjacent pixels, this algorithm can remember the deleting pixels' importance-degree to a certain extent. The article [11] achieves the goal that maintain the image characters by adjusting the seams that pass throuth the trait points in image. Futhermore, The paper [12-19] also enhance the seam carving algorithm.

Within all the above seam-carving algorithms, the Backward way [1]'s status is indisputable, its advancement and practicability are more superior than other methods.

\section{The Image Resizing on Account of Deformation}

The image retargeting on account of deformation [2, 4-7, 20-27] is another efficient method with respect to content-aware image resizing. Firstly, this algorithm segments the image into meshes, then within the limitation of target image displayer's size, maintains the vital meshes with higher importance-degree unchanged(or vary these meshes with equal ratio resizing) and lets the background areas with lower importance-degree stretched, then generates the refreshing meshes, finally, applying the inverse mapping of corresponding meshes can produce the target picture.

In 2008, Wang has presented a optimized scale-and-stretch image retargeting method(SNS)[7] at the Siggraph Asia. Firstly, this algorithm segmented the image into meshes $(V, E, F), V$ is called as the vertex set, $E$ is regarded as the border set, and $F$ is known as the mesh set. $V=\left[v_{0}^{T}, v_{1}^{T}, \cdots v_{e n d}^{T}\right]$ and $v_{i} \in R^{2}$ point out the primal vertex location. For the sake of resizing the $m \times n$ picture to the arbitrary sizes of $m^{\prime} \times n^{\prime}$, we can fix the topleft corner vertex $v_{0}$ immovability and ask the user appoint the new location to the belowright corner vertex $v_{\text {end }}$. Other vertexes on the border slip along the border under the rectangle restriction. We have found the troubles in deformation meshes 
$V^{\prime}=\left[v_{0}^{\prime^{T}}, v_{1}^{\prime^{T}} \cdots v_{\text {end }}^{\prime^{T}}\right]$. With respect to each $f \in F$ about shape variables, SNS method calculates the distance that is between deformed mesh $v^{\prime}$ and original mesh $v$ 's uniform retargeting version $s_{f} v+t\left(s_{f}\right.$ is the retargeting parameter, $t$ is the amount of movement), for the sake of measuring the mesh's shape variables $D_{u}(f)$ :

$$
D_{u}(f)=\sum_{\{i, j\} \in E(f)}\left\|\left(v_{i}^{\prime}-v_{j}^{\prime}\right)-s_{f}\left(v_{i}-v_{j}\right)\right\|^{2}
$$

Finally, each mesh deformation's influence to all the meshes' deformation $D_{u}$ has something to do with corresponding mesh's importance-degree $w_{f} \cdot w_{f}$ is known as the average value of mesh's pixels. Hence the all the meshes' total deformation $D_{u}$ is defined as: $D_{u}=\sum_{f \in F} w_{f} D_{u}(f)$.

According to the fact that most main objects usually take over the continuous meshes in the image, SNS algorithm maintains the main object's character through minimizing the mesh border lines' bending energy. This method is maintaining the orient of border and adjusting its length. SNS algorithm also regarded the mesh line's bending energy as:

$$
D_{l}=\sum_{\{i, j\} \in E}\left\|\left(v_{i}^{\prime}-v_{j}^{\prime}\right)-l_{i, j}\left(v_{i}-v_{j}\right)\right\|^{2}, l_{i, j}=\left\|v_{i}^{\prime}-v_{j}^{\prime}\right\| /\left\|v_{i}-v_{j}\right\|
$$

Ultimately, we should minimize the sum of meshes' deformation value and mesh lines' bending energy that is described by $D$ :

$$
D=D_{u}+D_{l}
$$

During the computation process, at the first step, SNS algorithm speculates the mesh vertex $v^{\prime}$, calculates excellently the mesh's retargeting parameter $s_{f}$, then under the constraint size of the displayer border updates the mesh vertex $v^{\prime}$, achieves goal iteratively at last.

After you have divided the image into grid meshes employing the SNS algorithm, it is obvious to generate the meshes that occupy main object and background areas, which can not realize the coincidence with mesh vertexes and image main object feature, and isn't conducive to the keep of local characters. To address this problem, the reference [23] recognizes the border's feature points that pass through the main object, connects feature points in the mesh with other feature points in order to generate the lots of controlling nodes, finally keeps the image's feature by optimizing controlling nodes. The reference [24] has improved the article[23]'s optimizing function, which avoids the deformation in more important areas.

The paper [2, 22] have much similarity to SNS algorithm, which also segment the image into meshes. When representing the resizing ratio about meshes, it isn't same as SNS algorithm which employs the semblable resizing parameter $s_{f}$, but distinguish the retargeting parameter $a_{i, j}^{1}, a_{i, j}^{2}$ with respect to $\mathrm{x}, \mathrm{y}$ axis. Next according to importance-degree in mesh, adjusting $r=a_{i, j}^{1} / a_{i, j}^{2}$ the value of approach to 1 for the sake of controlling the corresponding mesh's deformation value. About meshes with higher importance degree, we 
try our best to adjust the $\mathrm{r}$ close to 1 . But with regard to lower degree mesh, we make the $\mathrm{r}$ depart from 1 in order to achieve the target displayer's size.

The method and mesh energy in article [2, 22]is similar to each other, the primary difference between them is the solution to optimizing the mesh energy. The article [2] optimizes the single mesh energy partially in iteration to settle the retargeting factor $a_{i, j}^{1}, a_{i, j}^{2}$, hence solves the mesh points' location by the entire optimization about the sum of all the meshes, this algorithm is known as LG(local-Global) way. But the reference [22] employ the convex quadratic program algorithm, this method is regarded as QP method.

Effectively, SNS method [7] generates better effect that maintains the main object's construction in image by using the strategy of keeping the scaling parameter equally. But this algorithm doesn't think over that different scaling parameters may generate different effect to meshes, so the main object in result image retargets along with the original image retargeting, for example in result image the beerhouse (as shown in Figure 2) retargets much smaller so as to imbalance to other construction's ratio, and influences the entire vision effect. Due to QP algorithm employs the local-total optimized method to generate result, the consequence can not get the total optimization when getting the local optimization, and the effect in result is not stable. For example the beerhouse has been stretched much more but not keep the primary ratio(as shown in Figure 2). QP algorithm[22] employ the convex quadratic method to settle the entire resolution and get the better effect(Figure 2) by increasing the energy which forbids the meshes fold and amplifies the important areas.

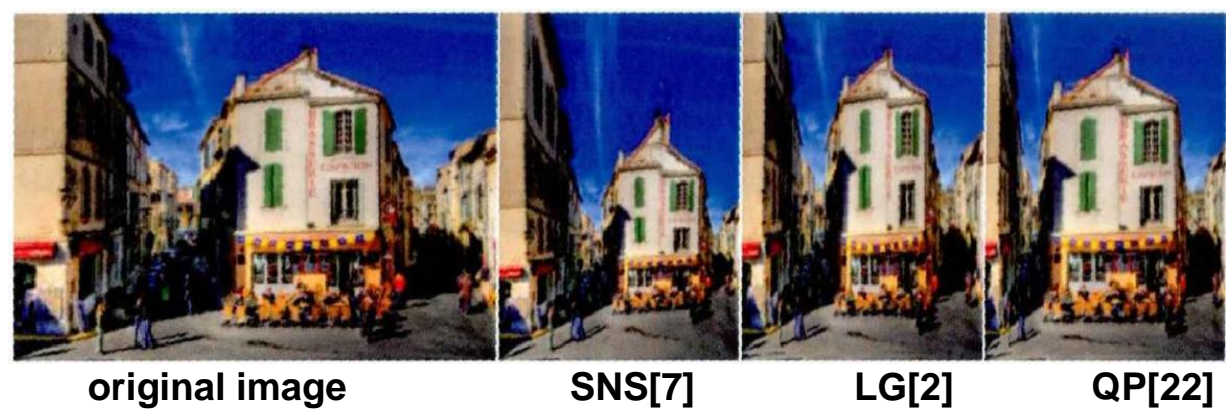

Figure 2. The Retargeting Result on Account of Image Deformation (reference[22])

\section{Multi-operator Retargeting}

Multi-operator retargeting [28-31,33] is a combo of seam- carving, conventional scaling and cropping, or merely the first two methods combo for the sake of realizing the contentaware image retargeting. A key problem in this algorithm is how to determine the operations' order and amount. A straightforward way is that shows all the results of different operations' amount and order, then selects the optimal one. If allowing reducing and enlarging be intersection cross, the types of algorithm is infinite. Therefore when reducing (enlarging) the picture, firstly we should set up every operation merely reducing(enlarging) course. Under this premise, if employing $m$ operations to transform the picture size $w$, then the operation types are $m^{v}$, it is very big, so current multi-operator algorithms usually have some additional assumption to reduce the amount of calculation. The commonly used assumptions have been segmented into two forms: one of these is to find regular route[28], based on the fact that the operation amount's influence is more prominent than the order's, fixing the order can reduce the calculation to search the optimal operation amount; The other form is 
searching the mix route[28], firstly deciding each sub-operation amount $k(k \geq 1)$, then determining the operation order length $n$, finally employing dynamic programming algorithm to choose each operation's pattern. In addition, when we are looking for the optimal operation pattern, we merely reserve the optimum value about each type that has uniform operation amount but diverse order for the sake of reducing the calculation. If there are two types of operation in multioperator process, the method to searching the route shows in the Figure 3:

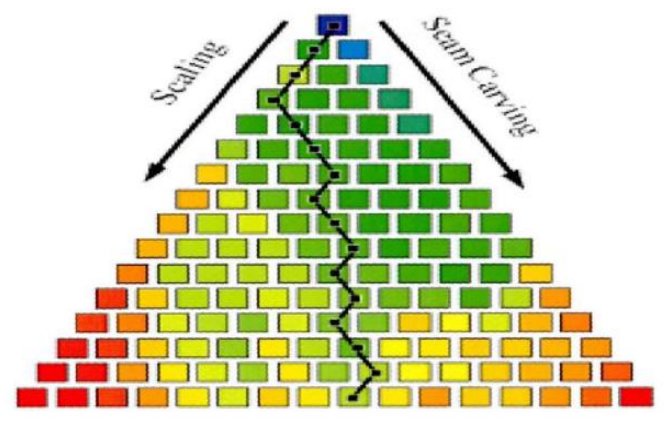

\section{Figure 3. Employing Dynamic Programming to look for the Optimal Mixed Route that only has Two Types of Operations [28]}

After determining the method to looking for operations' amount and order, a key problem to this technology is how to project a good measure criteria to measure similarity between the resized image and original, the main difference among all the multi-operator algorithm is also the measure criteria.

In 2009, Rubinstein presented a multi-operator method[28] at the SIGGRAPH. The paper indicated that multi-operator's effect is better than only single operation's result, because this text analyzed statistically towards customer satisfaction to results that is generated by different algorithms (single seam-carving, cropping, traditional scaling, or the combo of three operations).

Rubinstein's multi-operator [28] algorithm employed Bi-Directional Warping(BDW) in order to measure the difference between the target image with original. BDW contains the similarity between resized image with original image(representing the coupling with resized and original, in other words, how much noise in resized image that was taken by retargeting original image) and the similarity between the resized image with original(representing the integrality that resized image to original image content). Firstly, BDW segments the original image into blocks, then looking for the most similar block within the original image, which is used by dynamic programming algorithm. The method of searching for optimal matching block applies the consistent between the matching blocks along with the original order, and only permits several-for-one not one-to-several. Finally, the average color value of all the blocks' pixels is computed and regarded as the discrepancy between the images (as shown in Figure 4). On the basis of the discrepancy value, the article [28] employs the regular route and mixed route respectively, looks for the optimal operation sequence and gets the corresponding result. 

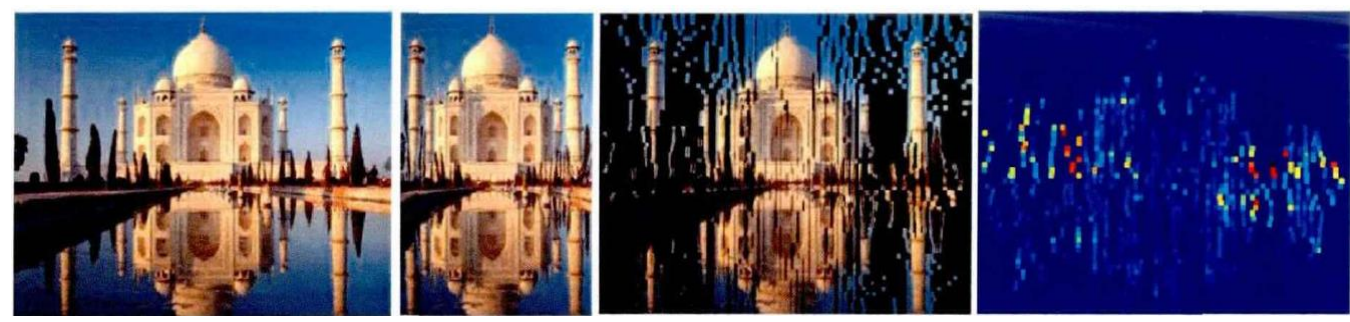

original image(S resized image $T \rightarrow S$ matching blocks $L 1$ distance between the blocks

Figure 4. Applying BDW Method to Look for the Optimal Matching Blocks [28]

\section{The Qualitative Analysis and Comparison of Three Kinds of Resizing Techniques}

This paper gives the qualitative analysis and comparison of three kinds of resizing techniques from the eight items below, the reader can experience the following items from Figure 5 :

Item 1: From the view of the nature of the problem's solving, seam carving for pixel processing only produces two kinds of results, one is duplicatting pixels to enlarge image, the other is narrowing or deleting pixels to reduce the image, which belongs to discrete switch operation, the properties of its solution is discrete; And image deformation method(SNS) based on processing grids, by stretching to all pixels in the grids within the whole tensile deformation to achieve the purpose of resizing in, its changing for the operation of the pixels is a continuous way, thus with respect to algorithm based on the deformation, its solution method is a continuous domain algorithm; Retargeting method based on multiple operating, due to it is combination of seam carving, cropping and uniform scaling of a variety of simple operations, so we can analyze according to these simple operations, among them, seam carving and cropping of the solution are a discrete domain, and the unified scaling method for solution properties belongs to the continuous domain. For the multi-operation, its simple operations both belong to the discrete domain and the continuous domain, so the nature of the multi-operation to solve the problem is not belonging to the continuous domain, also do not belong to the discrete domain, it is a complex of continuous and discrete operation. 

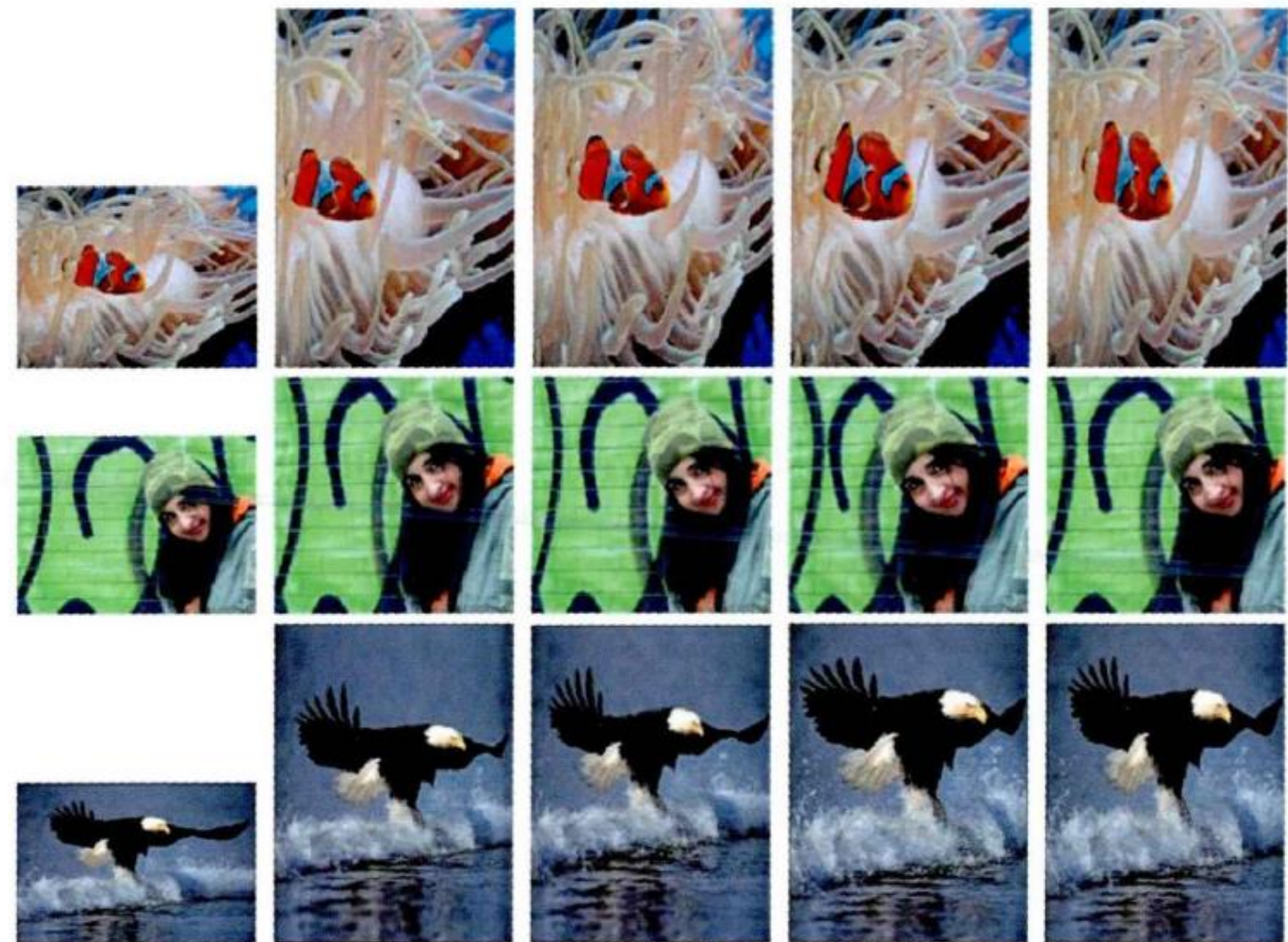

SNS[7]

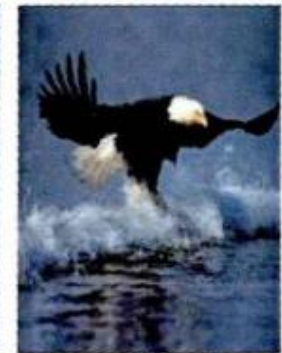

L/G[2]
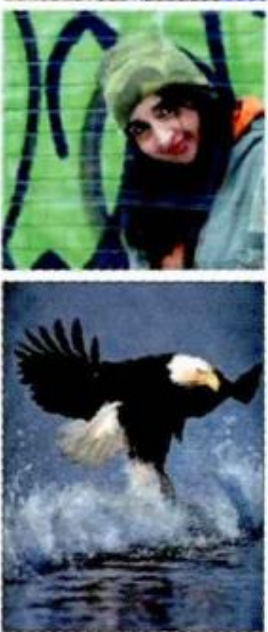

$\mathrm{SC}[1]$
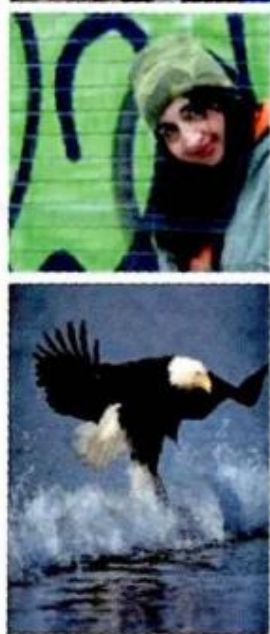

MOP[30]

Figure 5. The Results of Different Resizing Methods [32]

Item 2: From the view of the dependence of Importance-Map about the three methods, three methods involved in this paper all belong to the content-aware image resizing method. The core of content-aware resizing is, as far as possible makeing the important areas of high important degree invariability, and making the deformation produced in the unimportant areas. Here, how to measure the important degree about the image areas? Our basis is Importance-Map. If there is not Important-Map, content-aware image retargeting technology is impossible, therefore, no matter the above three algorithms use different ways, as long as they belong to the category of content-aware technology, all need to the graph based on the important degree, which leading to different resizing technology. So, three ways need to Importance-Map.

Item 3: For homogeneous areas in background, sometimes they were referred to as the redundant information, their ways of treatment and utilization to themselves are still different. Three ways have their own characteristics. Look at the seam-carving method, recalling seamcarving, you can see, when changing the image width (height), we use the redundant information that is vertical (horizontal) seams, that is to say, we use the redundant information which is vertical with changing direction; And algorithms based on the deformation of the image resizing, first divide the image into the grids, the gird may be a rectangle, triangle, trapezoid, which is various and flexible. When we change the redundant information, for redundant information grid we can scale, stretch, and enlarge in any direction, so algorithm based on the deformation of the available redundancy information, there is no special requirements on direction; Multi-operation method, is made by the operation simple ways of seam-carving and uniform scaling. This is very easy to see, the 
direction requirement of the seam-carving method for redundant information, which is very high, but the uniform scaling way in the direction of the redundant information should not have strict requirements, so multi-operation way of dealing with redundant information is also very flexible and can use the redundant information that should not have special requirements for direction.

Item 4: Whether to produce too much artificial noise? No matter any resizing way, they should produce artificial noise inevitably, because we make the operation for the original image's transformation in size, so the noise is inevitable. In this paper, the three ways are more or less in the production of the artificial noise. So which method produces more noise about these methods, which generates less? According to the nature of the resizing methods, This may get the answer: based on the operation of the seam-carving is discrete, the pixel processing result is retaining, or copying, this simple operation is a double-edged sword, handling method of the operation is received easily, and algorithms are easy to achieve, but excessive seams could generate information loss, this is a more serious problem than artificial noise. Therefore, seam-carving sometimes can be applied to object deleting; Based on image deformation operation, because its solution is the nature of the continuous domain, so for the information processing of the original image, which is more smooth, more natural transition, and the users are easy to accept the effect. Colour changing of applying this algorithm is more continuous, so the artificial noise of deformation method is relatively smaller than the seamcarving; After understanding the two algorithms of the relationship between solutions' nature and artificial noise, based on the multi-operation's artificial noise that readers can infer. Due to the multi-operation way is in the middle of discrete and continuous operation, so the amount of artificial noise that is produced by the multi-operation is also in the middle of the seam-carving and deformation.

Item 5: The third item analyzes the redundant information above, the purpose of processing the redundant information is to keep the main object information, we compare the three ways in terms of the deformation degree of main object. When seam carving algorithm solves problems, it removes the seam of minimum energy. In general, the pixels of main object, rarely in the seam, so whatever for operating seams (delete or copy), essentially we deform information of the main body rarely, or almost don't change (because the pixels in the main object are almost nonexistent in seams); Based on the deformation way, divide the image into the grids firstly, then discriminate grids according to the important degree of the grids, and applies different stretching method. Attention, when algorithm stretches homogeneous background area more excessively, the main object grids may be more or less stretched, because, after all, all meshes should keep the rectangular shape of the original image on the whole. So the stretching degree of deformation algorithm for main object is the largest, this is because this approach should consider deformation to meet the rectangular constraints of the image on the whole; So it is easy to get, for main object's deformation extent, multi-operation is the way combination of seam-carving and SNS, also because it is a joint of single operations, so its characteristics (whatever advantages disadvantages) are not the most prominent, for main object's deformation degree is inferior to seam-carving, a bit better than the SNS, is the middle level.

Item 6: Any features of algorithm are essentially comes from the nature of the solution, in view of the three resizing methods from the nature of the solution we can analyze the question of the angle of content losing, seam carving for pixels is discrete operations (sometimes known as switch operation), which inevitably produces content losing, because every operation is set for pixels (seam) to delete or copy, so content loss is inevitable. The method based on the deformation, for the operation of the pixels, from the overall grasp of the 
processing of different grid, although such operation may also generate content losing, but its solution for content is the continuous operation, is gentle, and can't be as simple as seam carving, so based on deformation algorithm for image content reservation is much better than seam carving. So multi-operation algorithm, because it includes seam carving operation, so its effect to keep object content inferior to SNS method. And because it is not just the seam carving operation, seam-carving is only a kind of its operation, in addition to this, and contains the uniform scaling, so for the contents of the retaining multi-operation is better than pure seam-carving.

Item 7: On the speed of the algorithms above, whether based on seam-carving, or based on image deformation operation, they are belonging to a single operation algorithm, so the operation speed is faster than the multi-operation. But multi-operation should not only considers the number of sub-operation, but also considers the sequence of sub-operation, its involved problem is complicated, and all the multi-operation method are carried out under certain assumptions, the purpose is to improve the computing speed, increase operational efficiency. While satisfying the demanding assumptions, multi-operation in speed is not satisfactory, so within the three methods proposed in this paper, multi-operation method in speed is the slowest.

Item 8: The three ways putted forward in this paper are difficult to get the comparison of the advantages and disadvantages simply, we can only say that each method has its characteristics and the scope of adaptation. The suitable image for seam-carving is: when smooth background areas' orientation in the image is vertical with the size of the image changing direction, employing the seam-carving can obtain better effect, but it is worth noting that the scaling ratio should not be too big, because of excessive applying of seams may destroy the proportion of the overall structure of the original image, and even delete the main object; The method based on the deformation of adaptive image has the following features: the subject and the background contrast intensely, main texture is complex, and background texture is simple; When multi-operation resizes the image, firstly applying seam-carving method, as mentioned above, seam-carving could introduce artificial noise, so when the seam-carving generate artificial noise too much, multi-operation will be transferred to other operation to avoid more artificial noise, so multi-operation of adaptive image is: when introducing too many artificial noise after emplying the seam-carving, this algoritm will turn into more than other single operation ways to deal with the images.

Finally, this article summarizes the above 8 items in the table, such as Table 1:

Table 1. The Qualitative Comparison of Three Ways

\begin{tabular}{|c|c|c|c|}
\hline Character & Seam-carving & Deformation(SNS) & $\begin{array}{c}\text { Multi- } \\
\text { operation }\end{array}$ \\
\hline Nature of the solution & Discreteness & Continuity & $\begin{array}{c}\text { A combination of } \\
\text { discreteness and } \\
\text { continuity }\end{array}$ \\
\hline Importance-Map & Need & Need & Need \\
\hline $\begin{array}{c}\text { Available redundant } \\
\text { information }\end{array}$ & $\begin{array}{c}\text { A direction perpendicular to } \\
\text { the direction of size } \\
\text { changing }\end{array}$ & $\begin{array}{c}\text { Both horizontal } \\
\text { and vertical directions }\end{array}$ & $\begin{array}{c}\text { Both horizontal and } \\
\text { vertical directions }\end{array}$ \\
\hline Aanual noise & More & Less & Middle \\
\hline $\begin{array}{c}\text { Stretching of main } \\
\text { object }\end{array}$ & Less & More & Middle \\
\hline
\end{tabular}




\begin{tabular}{|c|c|c|c|}
\hline Content lossing & More & Less & Middle \\
\hline Speed & Fast & Fast & Slow \\
\hline $\begin{array}{c}\text { Characters of adaptive } \\
\text { image type }\end{array}$ & $\begin{array}{c}\text { Smooth background area } \\
\text { perpendicular to changing } \\
\text { direction of the image; } \\
\text { Resizing ratio is small }\end{array}$ & $\begin{array}{c}\text { Main subject and background } \\
\text { contrast intensely; The main } \\
\text { object has complex texture } \\
\text { and simple background } \\
\text { texture }\end{array}$ & $\begin{array}{c}\text { Seam-carving } \\
\text { introduces a lot of } \\
\text { artificial noise }\end{array}$ \\
& & & \\
\hline
\end{tabular}

\section{Conclusion}

In a word, until now there is no subjective or objective measure standard that assess the diverse retargeting ways' performance. Therefore, this paper makes some qualitative analysis with respect to three retargeting algotithms' character. Firstly, deformation algorithm and seam carving are more rapid than the multi-operator, especially than multi-operator on account of block-matching. Secondly, for result's performance, each method has its unique advantage and fitted image, there is no method which is universal to any types of image.

The characters of the seam-carving algorithm comes from the operation belonging to pixels. Due to the pixels, the resizing results are precise; Because the operation is discrete, the color retargeting result is most similar to original image. Similarly, due to the operation type is discrete, so the resizing result's structural information is also easy to be destroyed, and may produce artificial noise. Therefore, seam carving suits the image which has smooth background and less structural information, besides, seam carving algorithm also can remove the object.

The nature of the multi-operator is that balances the main objection's transformation, structure destroying, artificial noise and losing of image content, and then compromises. Currently, better operating method is that using seam carving for image firstly, while the seam-carving method destroys the image structure, generates more artificial noise or removing the background content that affecting the total visual quality, then gives up the seam-carving and employs scaling uniformly. In this algorithm, the continuity of scaling may maintain ratio of the image, and avoid the artificial noise. However, most of the multioperator ways in resizing process cannot look for the most optimal effect, and only generate better effect. Therefore, there is a feeling that multi-operator algorithm is not the most perfect method but a better one.

The deformation algorithm of retargeting image, which is limited by content important degree and border. Meanwhile, this method has continuity of scaling and keeps the traits of main object in image, can obtain retargeting factors of different meshes by optimizing the sum energy of meshes. However, when the meshes degenerate to a line or a point, the excessive compression to corresponding areas may generate content's discontinuity in image.

\section{Acknowledgment}

Thanks to the many references papers' authors whose images were used in this paper. We also thank the anonymous reviewers and referees for their comments.

\section{References}

[1] S. Avidan and A. J. Shamir, "ACM Transactions on Graphics", vol. 3, no. 26, (2007).

[2] Z. Karni, D. Freedman and C. Gotsman, Computer Graphics Forum, vol. 5, no. 28, (2009).

[3] M. Rubinstein, A. Shamir and S. J. Avidan, ACM Transactions on Graphics, vol. 3, no. 27, (2008).

[4] L. Feng and M. Gleicher, "Automatic Image Retargeting with Fisheye-View Warping", Proceedings of the 18th Annual ACM Symposium on UIST, New Youk, USA, (2005). 
[5] J. Shi, Y. Guo and Z. Du, Journal of software, zk, vol. 19, (2008).

[6] Y. Guo, F. Liu and J. Shi, IEEE Transactions on Multimedia, vol. 5, no. 11, (2009).

[7] Y.-S. Wang, C.-L. Tai, O. Sorking and T.-Y. Lee, ACM Transactions on Graphics, vol. 5, no. 28, (2009).

[8] L. Zhi, Y. Hongbo and S. Liquan, Optical Engineering, U.S.A., vol. 1, no. 49, (2010).

[9] Y. Pritch, E. Kav-Venaki and S. Peleg, "Shift-Map Image Editing", Proceedings of the Twelfth IEEE ICCV, Florida, USA, (2009).

[10] C. Sunghyun., C. Hanul and Y. Matsushita, "Image Retargeting Using Importance Diffusion”, Proceedings of the ICIP, Florida, USA, (2009).

[11] P. Deng, "Degree paper", Zhejiang University Press, Hangzhou, (2008).

[12] N. Hill and P. Eslambolchilar, "Seam carving for enhancing image usability on mobiles", Proceedings of the 22nd British HCI Group Annual Conference on HCI, Liverpool John Moores University, UK, (2008).

[13] R. Nakashima, K. Utsugi and K. Takahashi, IEICE Transactions, vol. D(6), no. 94, (2011).

[14] J.-W. Han, K.-S. Choi and T.-S. Wang, "Wavelet based seam carving for content-aware image resizing", Proceedings of the International Conference on Image Processing, Cairo, Egypt, (2009).

[15] J. Chen, L. Miao and X. Liu, J. World Wide Web, vol. 3, no. 14, (2010).

[16] A. Mansfield, V. Peter and Gehler, "Scene Carving: Scene Consistent Image Retargeting", Proceedings of the 11th European Conference on Computer Vision, Heraklion, Crete, Greece, (2010).

[17] C. Sunghyun, C. Hanul and Y. Matsushita, "Image Retargeting Using Importance Diffusion", Proceedings of the ICIP, Florida, USA, (2009).

[18] J. Scott, R. L. Tutwiler and M. Pusateri, "Hyper-spectral content aware resizing", Proceedings of the 37th IEEE Applied Imagery Pattern Recognition Workshop, Washington, DC, USA, (2008).

[19] A. Srivastava and K. Kishore Biswas, "Fast Content Aware Image Retargeting", Proceedings of the Sixth Indian Conference on Computer Vision, Graphics \& Image Processing, Bhubaneswar, India, (2008).

[20] T. Ren, H. Liu and G. Wu, Journal of software, vol. 0, no. 21, (2010).

[21] R. Gal, O. Sorkine and D. Cohen-Or, "Feature aware texturing", Proceedings of the Eurographics Symposium on Rendering, Goslar, Germany: Eurographics Association, (2006).

[22] C. Renjie, D. Freedman and Z. Karni, "Content-Aware Image Resizing by Quadratic Programming", Proceedings of the 2010 IEEE CVPR WorkShop on NORDIA, Florida, USA, (2010).

[23] G.-X. Zhang, M.-M. Cheng and S.-M. Hu, "Computer Graphics Forum Special issue of Pacific Graphics", vol. 7 , no. $28,(\mathbf{2 0 0 9})$.

[24] L. Lei, Journal of computers, vol. 10, no. 33, (2010).

[25] H. Bao and X. Li, "Non-uniform Mesh Warping for Content-Aware Image Retargeting", Proceedings of the 8th International Conference on Image Analysis and Recognition, Burnaby, BC, Canada, (2011).

[26] P.-Y. Laffont, J. Yun Jun and C. Wolf, "Interactive content-aware zooming", Proceedings of the Graphics Interface 2010 Conference, Ottawa, Ontario, Canada, (2010).

[27] S.-F. Wang and S.-H. Lai, "Fast structure-preserving image retargeting", Proceedings of the IEEE International Conference on Acoustics, Speech, and Signal Processing, Montreal, Canada, (2009).

[28] M. Rubinstein, A. Shamir and S. Avidan, ACM Transactions on Graphics, vol. 3, no. 28, (2009).

[29] W.-M. Dong and J.-C. Paul, “Adaptive content aware image resizing”, LIAMA, Tech. Rep., (2009).

[30] W.-M. Dong, N. Zhou and J.-C. Paul, ACM Transactions on Graphics, vol. 5, no. 28, (2009).

[31] W.-M. Dong, X.-P. Zhang and N. Zhou, "Fast multi-operator image resizing", LIAMA, Tech. Rep., (2009).

[32] M. Shi, Degree paper, Yunnan University Press, Kunming, (2011).

[33] D. Simakov, Y. Caspi, and E. Shechtman, "Summarizing visual data using bidirectional similarity", Proceedings of the IEEE Conference on CVPR, Florida, USA, (2008).

[34] K. Johannes, S. Ariel and P. Pieter, ACM Transactions on Graphics, vol. 6, no. 32, (2013).

[35] S. Goferman, L. Zelnik-Manor and A. Tal, IEEE Transactions on Pattern Analysis and Machine Intelligence, vol. 10, no. 34, (2011).

[36] Y. J. Niu, F. Liu and M. Gleicher. J. Multimedia Tools Application, vol. 3, no. 56, (2012).

[37] J. Kopf, A. Shamir and P. Peers. J. ACM Transactions on Graphics (TOG), vol. 6, no. 32, (2013)

[38] M. J. Dahan, N. Chen, A. Shamir and D. Cohen. J. The Visual Computer, vol. 12, no. 28, (2012)

[39] A. Shtof, A. Agathos, Y. Gingold, A. Shamir and D. Cohen. J. Computer Graphics Forum, vol. 22, no. 32, (2013). 


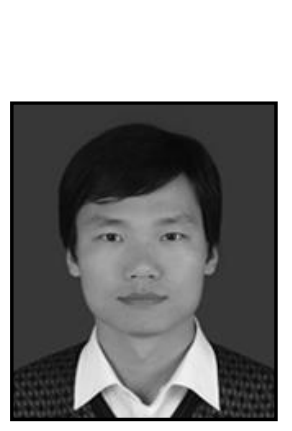

\section{Authors}

Zijuan Zhang, he received his B.S. degree in electronic engineering from Xi'an University of Posts and Telecommunications, China in June 2005 and his M.S. degree in mathematics from Shaanxi Normal University, China in June 2008. He is currently working towards his D.E. degree in Computer software and theory at Northwest University, China. His current research interest includes image and graphics processing.

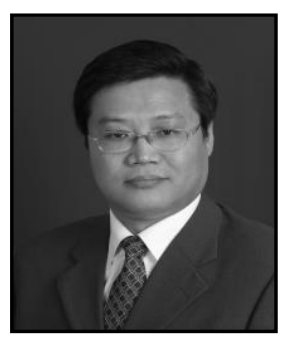

Baosheng Kang, he received his B.S. degree and M.S. degree in mathematics from Northwest University, China in 1982 and 1985. Then he received his D.E. degree in Computer science from Northwestern Polytechnical University in 1991. His current research interest includes image processing, image retrieval and computer graphics.

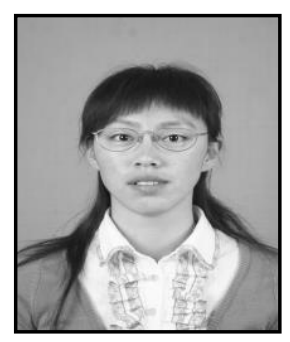

Shunye Wang, she received her B.S degree and M.S in Computer Science and Technology, Computer Applications from China University of Petroleum, China, in 2003 and 2006. Since 2006 she works in Langfang Teachers University. She hosts and participates in many projects, writes scientific papers. Her current research interest includes data mining and image mining. 
International Journal of Signal Processing, Image Processing and Pattern Recognition Vol.7, No.2 (2014) 